\title{
Orbital Arteriovenous Fistula around the Optic Nerve Treated by Transvenous Embolization
}

\author{
Masayuki SATO, Yuji MATSUMARU, Tatsuo AMANO, Yuki KAMIYA \\ Toranomon Hospital, Department of Endovascular Neurosurgery, Tokyo, Japan \\ Corresponding author: Masayuki SATO marchin16@yahoo.co.jp
}

\section{ABSTRACT}

Intraorbital arteriovenous fistula (AVF) is a very rare disease; therefore an optimal treatment strategy has not yet been established. Here we describe the use of high-resolution cone-beam computed tomography (CBCT) and selective angiography to visualize the detailed anatomy of orbital vessels. The information provided by the intraoperative imaging led to a change in treatment strategy and eventually to successful transvenous coil embolization. A 55-year-old man presented with a 4 months history of right exophthalmos, chemosis and diplopia. Magnetic resonance (MR) imaging demonstrated a dilated superior orbital vein. Cerebral angiography showed an intraorbital AVF fed by the branch of the ophthalmic artery and drainage to the superior orbital vein (SOV) and inferior orbital vein (IOV). Superselective angiography and high-resolution CBCT with diluted contrast medium clarified that feeders of the AVF came from the first segment of the ophthalmic artery, and the shunt located surrounding an optic nerve sheath. Transvenous embolization via IOV with coils occluded the AVF. Detailed analysis of intraorbital vessels using high-resolution CBCT and selective angiography led to successful treatment by transvenous coil embolization.

KEYWORDS: Arteriovenous fistula, Embolization, Orbit, Superselective angiography

\section{INTRODUCTION}

I ntraorbital arteriovenous fistula (AVF) is a very rare disease and an optimal treatment strategy has therefore not been Lestablished yet $(3,5,7,8,10)$. Transarterial embolization with a liquid material carries a high risk because the blood supply to the retina and optic nerve is present within the orbit. In this case report, selective angiography of the ophthalmic artery and high-resolution cone-beam computed tomography (CBCT) (VasoCT, Philips Healthcare, Best, The Netherlands) showed the shunt point of the AVF and the detailed anatomy of the orbital vessel that related to visual function $(1,9)$. We report an effective and safe treatment for orbital AVF using the anatomical information obtained by modern imaging technology.

\section{CASE REPORT}

A 55-year-old male presented with right exophthalmos, chemosis and diplopia, and gradual worsening of right visual acuity. Neurological examination showed ptosis and reduced vision of the right eye. Magnetic resonance (MR) imaging and MR angiography demonstrated a dilated superior orbital vein (SOV), suggesting a cavernous sinus dural AVF (Figure $1 \mathrm{~A}-\mathrm{C}$ ). However, diagnostic cerebral angiography revealed an AVF in the right orbit fed by the branch of the right ophthalmic artery and draining to the right facial vein through the right SOV and inferior orbital vein (IOV) (Figure 2A, B). It also showed a choroidal crescent. Then, we performed high-resolution CBCT with $50 \%$ diluted contrast medium, which clearly indicated that the AVF was located in the orbit surrounding the optic nerve (Figure $3 \mathrm{~A}-\mathrm{C}$ ). It also unambiguously showed the central retinal artery near the eyeball (Figure 3B). Trans-arterial embolization by liquid material was therefore deemed too high risk as migration of the embolic material to the central retinal artery could lead to blindness. Consequently, we planned a transvenous approach via the facial vein. The jugular vein was cannulated with a 5 Fr Envoy guiding catheter (Codman,
Masayuki SATO (10): 0000-0001-8695-2966 Yuji MATSUMARU (D) : 0000-0002-4827-8410
Tatsuo AMANO (D): 0000-0003-3748-6684 Yuki KAMIYA (D): 0000-0003-3528-6953 

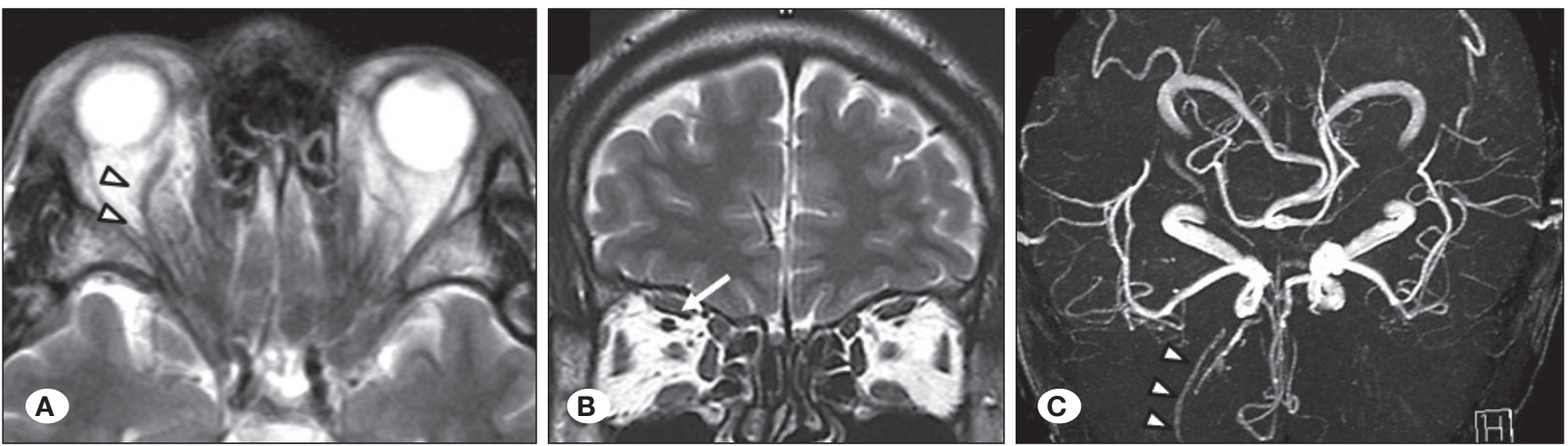

Figure 1: MR imaging (T2WI) and MR angiography of pre-embolization image (A-C). A) Dilated SOV (open arrow head), B) dilated SOV (white arrow), C) dilated SOV (open arrow heads).
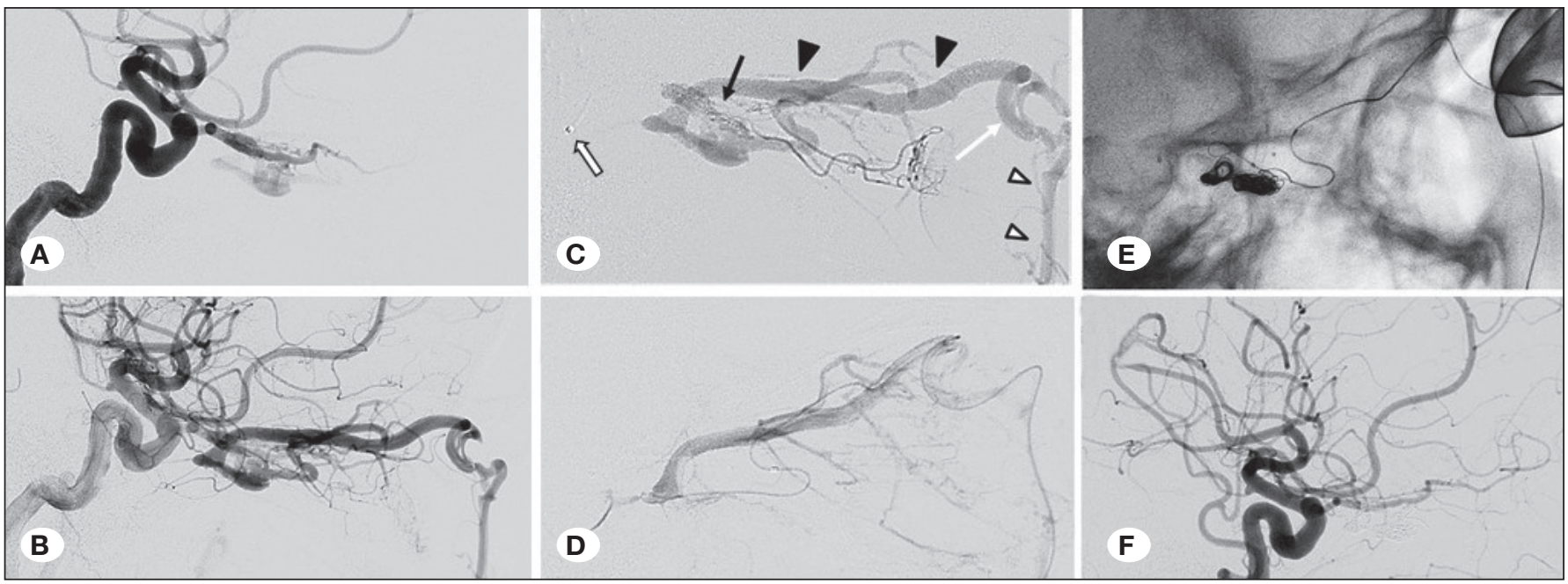

Figure 2: Angiography at the endovascular therapy. A, B) Selective angiography of left internal carotid artery (A: early phase, B: delayed phase). C) Selective angiography of left ophthalmic artery. Shunt point (black arrow), SOV (arrow heads), angular vein (white arrow), facial vein (open arrow heads), tip of microcatheter (open arrow) (Echelon 10 microcatheter, COVIDIEN, Mansfield, MA, USA), D) Selective venography (Echelon 10 microcatheter, COVIDIEN, Mansfield, MA, USA), E) inserted platinum coils (Trufill DCS Orbit, Cordis Neurovascular, Miami Lakes, FI, USA) and hydrogel coils (Hydrosoft, Terumo, Tokyo, Japan) and set a fulcrum from the body surface with a gloved finger of lead. F) Post-embolization left common carotid angiography (Lt.CCAG).
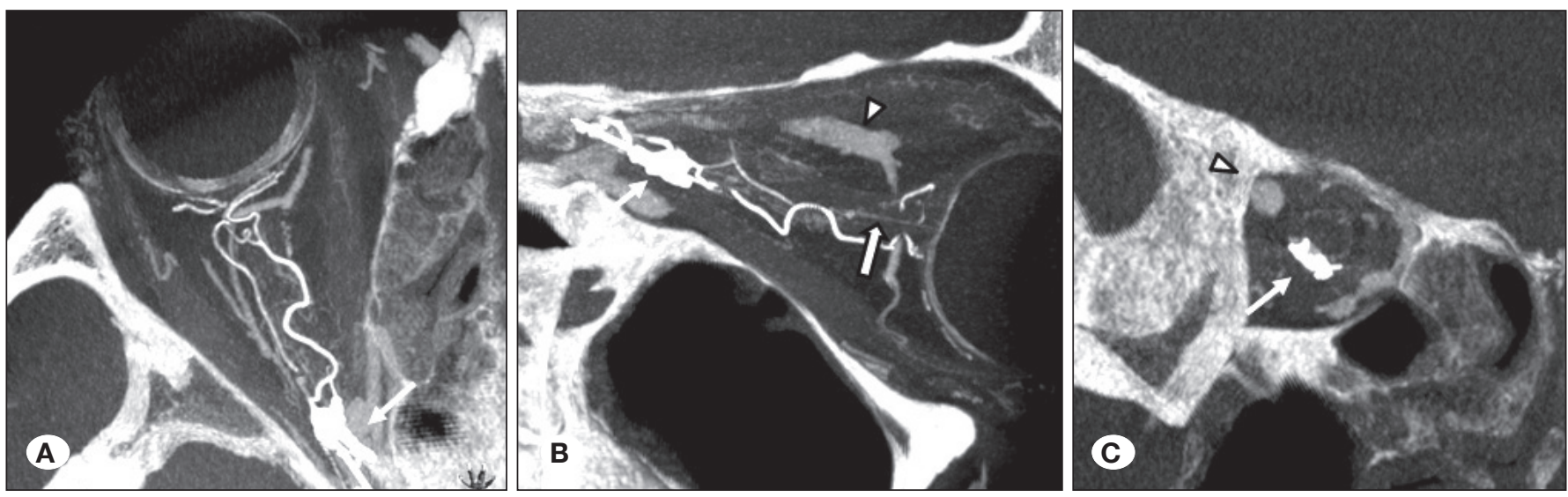

Figure 3: Cone beam computed tomography (A: axial image, B: sagittal image, C: coronal image) with diluted contrast medium from ophthalmic artery. Shunt point (white arrow), central retinal artery (open arrow), SOV (open arrow head). 
Raynham, Massachusetts, USA), an Echelon 10 microcatheter (COVIDIEN, Mansfield, MA, USA) with a CHIKAI 0.014 inch microguidewire (ASAHI INTECC, Tokyo, Japan) introduced to the shunting point with fulcrum by finger with lead gloves (Figure 2C, D). The placement of platinum coils (Trufill DCS Orbit, Cordis Neurovascular, Miami Lakes, FI, USA) and hydrogel coils (Hydrosoft, Terumo, Tokyo, Japan) (Figure $2 \mathrm{E}$ ) occluded the AVF completely (Figure 2F). Consequently, exophthalmos and diplopia also improved gradually after embolization.

\section{DISCUSSION}

There are some reports of intraorbital arteriovenous malformations (AVM) $(3,5,7,8,10)$. Symptoms and signs are the same as for cavernous sinus dural AVF (DAVF), namely chemosis, exopthalmos, loss of visual acuity, and extraocular motor palsy.

It is difficult to diagnose on any imaging modality whether intraorbital AVF is related to the AVM or DAVF. It can be considered as AVM because the optic nerve is a part of the central nervous system. There may be congenital AVM (2). On the other hand, the optic nerve in the orbit is carried with the optic sheath, which is connected with the inner layer of intracranial dura mater. Therefore, DAVF can even occur in this lesion. In this case, selective angiography and high-resolution CBCT indicated that the AVF presented in the dura mater surrounding the optic nerve sheath. Initial clinical symptoms of venous hypertension were at the middle age period, which also suggests that this shunt lesion was acquired and similar to DAVF. Therefore, we thought this shunt could be a DAVF.

Surgical removal is the most common treatment for orbital AVF (4). However, embolization is an alternative treatment option in selected cases. Other treatments are radiation therapy, percutaneous embolization, and laser therapy $(3,5,7,8,10)$.

The central retinal artery penetrates the optic nerve $1-1.5 \mathrm{~cm}$ behind the globe, and the course of the artery then becomes straight. The central retinal artery represents a terminal branch of the ophthalmic artery without well-established anatomical connections. The diameter of the central retinal artery is generally below $0.3 \mathrm{~mm}$, and hence it is difficult to visualize by angiography (6). For safe embolization, the microcatheter should be advanced beyond the second portion of the ophthalmic artery to prevent embolic material from entering the central retinal artery (6).

Cerebral angiography is still the golden standard for diagnosis, depiction of the vessel architecture, and treatment planning. More recently, high-resolution CBCT (VasoCT) has permitted visualization of intracranial stents during cerebral aneurysm embolization as well as the visualization of the detailed cerebral vascular anatomy in acute ischemic stroke $(1,9)$. In the present case, superselective angiography of the right ophthalmic artery showed the AVF, and VasoCT clearly described the AV fistula located on the orbit surrounding an optic nerve, which is very helpful for understanding the three-dimensional artery structure and the relationship between the shunt point and the optic nerve. The images showed that the shunt was located on the optic sheath and the feeder branched just proximal of the central retinal artery. This information suggested that transarterial embolization by liquid embolics carried a high risk of migration of embolic material to the central retinal artery, leading to total visual loss (6). Based on the information provided by the VasoCT scan, it was decided to change the treatment strategy and perform the embolization via a transvenous approach, which eventually led to a successful clinical result.

\section{CONCLUSION}

CBCT with diluted contrast medium is very important in evaluation of the detailed vessel architecture and the shunt point in orbital AVF. This method provides necessary information to determine the optimal treatment strategy for a successful coil embolization.

\section{REFERENCES}

1. Blanc R, Pistocchi S, Babic D, Bartolini B, Obadia M, Alamowitch S, Piotin M: Intravenous flat-detector CT angiography in acute ischemic stroke management. Neuroradiology 54: 383-391, 2012

2. Dayani PN, Sadun AA: A case report of Wyburn-Mason syndrome and review of the literature. Neuroradiology 49(5): 445-456, 2007

3. Deguchi J, Yamada M, Ogawa R, Kuroiwa T: Transvenous embolization for a purely intraorbital arteriovenous fistula. Case report. J Neurosurg 103: 756-759, 2005

4. Kaufman Y, Cole P, Dauser R, Hollier L Jr: Intraorbital arteriovenous malformation: Issues in surgical management. J Craniofac Surg 18: 1091-1093, 2007

5. Lin CJ, Blanc R, Clarencon F, Piotin M, Spelle L, Williams $\mathrm{M}$, Moret J: Transvenous embolization of an intraorbital arteriovenous fistula using Onyx. J Clin Neurosci 17: 783-785, 2010

6. Matsumaru $\mathrm{Y}$, Alvarez $\mathrm{H}$, Rodesch $\mathrm{G}$, Lasjaunias $\mathrm{PL}$ : Embolisation of branches of the ophthalmic artery. Interv Neuroradiol 3: 239-245, 1997

7. Naqvi J, Laitt R, Leatherbarrow B, Herwadkar A: A case of a spontaneous intraorbital arteriovenous fistula: Clinicoradiological findings and treatment by transvenous embolisation via the superior ophthalmic vein. Orbit 32:124126, 2013

8. Sato K, Matsumoto Y, Kondo R, Tominaga T: Intraorbital arteriovenous malformation treated by transarterial embolization: Technical case report. Neurosurgery 68: 383-387, 2011

9. Snoeren RM, Soderman M, Kroon JN, Roijers RB, de With $\mathrm{PH}$, Babic D: High-resolution 3D X-ray imaging of intracranial nitinol stents. Neuroradiology 54: 155-162, 2012

10. Subramanian PS, Gailloud PH, Heck DV, Tamargo RJ, Murphy KJ, Miller NR: Embolization of orbital arteriovenous fistulas. J Neurosurg 105: 797; author reply 797-798, 2006 